\title{
Mechanocatalytic Depolymerization of Cellulose With Perfluorinated Sulfonic Acid lonomers
}

\author{
Ayman Karam ${ }^{1,2 \dagger}$, Prince N. Amaniampong ${ }^{1 \dagger}$, José M. García Fernández ${ }^{3}$, Claudio Oldani ${ }^{4}$, \\ Sinisa Marinkovic ${ }^{5}$, Boris Estrine ${ }^{5}$, Karine De Oliveira Vigier ${ }^{1,2}$ and François Jérôme ${ }^{1,2 *}$ \\ ${ }^{1}$ INCREASE (FR Centre National De La Recherche Scientifique 3707), ENSIP, Poitiers, France, ${ }^{2}$ Institut de Chimie des Milieux \\ et Matériaux de Poitiers, Université de Poitiers, Centre National de la Recherche Scientifique, ENSIP, Poitiers, France, \\ ${ }^{3}$ Instituto de Investigaciones Químicas, CSIC - University of Sevilla, Sevilla, Spain, ${ }^{4}$ Solvay Speciality Polymers, Bollate, Italy, \\ ${ }^{5}$ ARD-Agro-Industrie Recherches et Développements, Green Chemistry Department, Route de Bazancourt, Pomacle, France
}

Here, we investigated that the mechanocatalytic depolymerization of cellulose in the presence of Aquivion, a sulfonated perfluorinated ionomer. Under optimized conditions, yields of water soluble sugars of 90-97\% were obtained using Aquivion PW98 and PW66, respectively, as a solid acid catalyst. The detailed characterization of the water soluble fraction revealed (i) the selective formation of oligosaccharides with a DP up to 11 and (ii) that depolymerization and reversion reactions concomitantly occurred during the mechanocatalytic process, although the first largely predominated. More importantly, we discussed on the critical role of water contained in Aquivion and cellulose on the efficiency of the mechanocatalytic process.

Keywords: cellulose, depolymerization, mechanocatalysis, Aquivion, biomass

\section{INTRODUCTION}

With the transition of our society to a more sustainable development, the manufacture of chemicals, and fuels from renewable feedstocks has become a priority (Huber et al., 2006; Corma et al., 2007; Dhepe and Fukuoka, 2008; Rinaldi and Schüth, 2009a,b; Bozell and Petersen, 2010; Climent et al., 2011; Van de Vyver et al., 2011; Zhou et al., 2011; Gallezot, 2012; Luterbacher et al., 2014; Yabushita et al., 2014; Wang et al., 2015). In this context, due to its low cost, large availability and non-edibility, cellulose, a biopolymer made of $\beta-1,4$ linked $\mathrm{D}$-glucose units, represents an attractive raw material. However, cellulose is highly recalcitrant to chemical processing due to the existence of a robust hydrogen bond network, both intrachain between glucose monomers in a single polymer strand and interchain between adjacent polymer chains, van der Waals interaction and electronic effects (Klemm et al., 2005; Shen and Gnanakaran, 2009; Siró and Plackett, 2010; Moon et al., 2011), which protect the glycosidic bond against hydrolysis, a pre-requisite step for the conversion of cellulose to soluble products. Hence, in many cases, the hydrolysis of cellulose requires high temperature or pressure, which leads to the concomitant formation of unwanted byproducts and thus to tedious work-up procedures.

Recently, mechanocatalytic depolymerization of lignocellulosic biomass has emerged as a contemporary frontier in biorefining (Zakrzewska et al., 2010; Groote et al., 2013; Zhang and Jérôme, 2013; Käldström et al., 2014; Kaufman Rechulski et al., 2015). This technology utterly overcomes the challenges and drawbacks posed by the recalcitrance of lignocellulose, in particular thanks to a synergistic effect between mechanical forces and catalysis (Beyer and ClausenSchaumann, 2005; Barraud et al., 2008; Carrasquillo-Flores et al., 2013). Although the use of milling for altering the behavior of cellulose has been an old-age practice, as well as the influence 


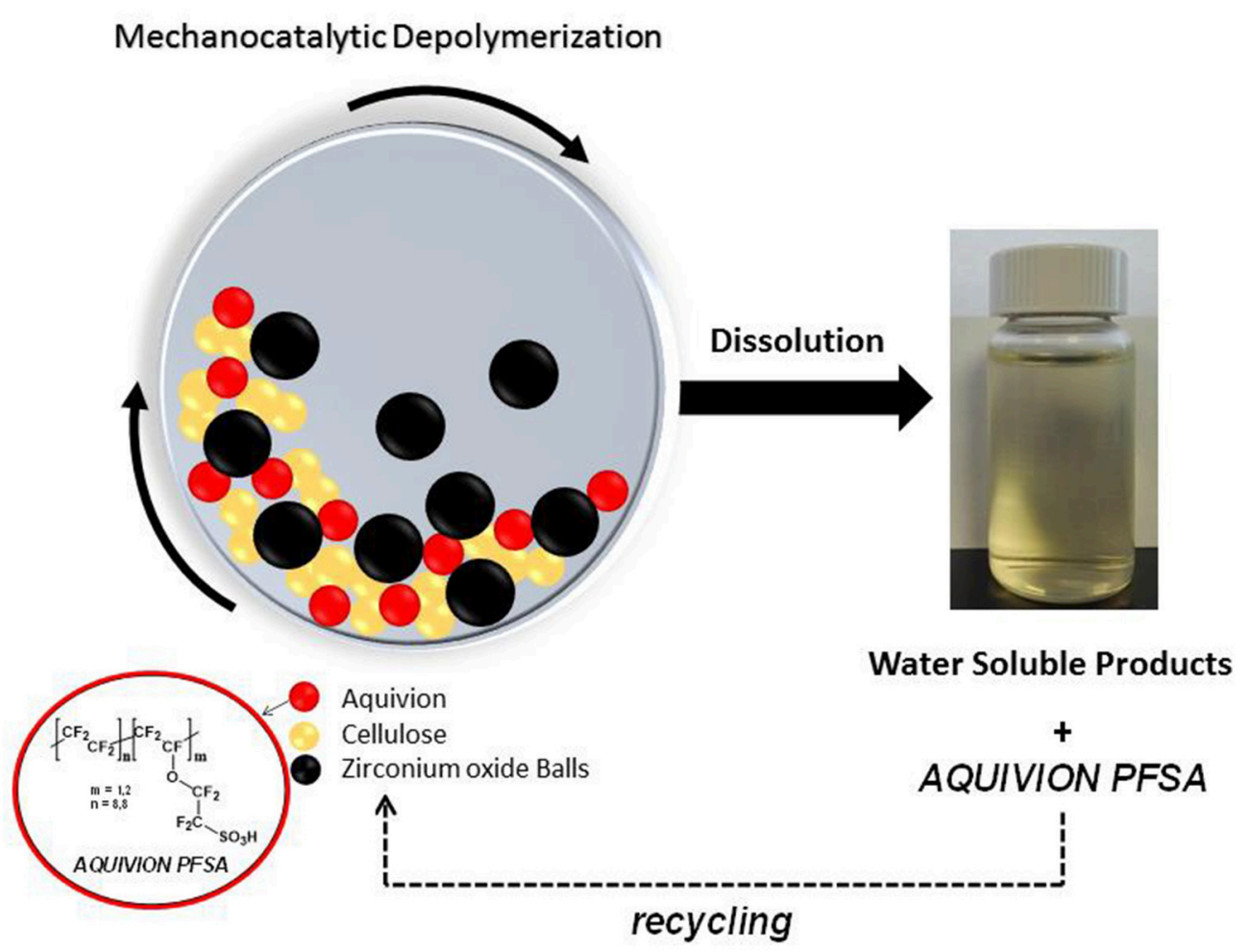

GRAPHICAL ABSTRACT | Aquivion PFSA, a sulfonated perfluorinated ionomer, is capable of depolymerizing selectively microcrystalline cellulose to oligosaccharide (DP up to 11).

of mechanical grinding on the reactivity of cellulose, the mechanocatalytic depolymerization of cellulose has garnered momentum in recent years (Hick et al., 2010; Meine et al., 2012). Mechanical forces provide energy to alter the crystalline structure and to reduce the particle size of cellulose. More importantly, it also changes the conformation of cellulosic chains and that of the glucose units, thereby lowering the exoanomeric effect responsible to a large extent for the high stability of the $\beta-1,4$ glycosidic bond in cellulose (Hick et al., 2010; Carrasquillo-Flores et al., 2013; Loerbroks et al., 2013; Schmidt et al., 2016). Hence, by combining mechanical forces and an acid catalyst, cellulose was depolymerized in a large extent to water-soluble products that can be further processed into other valuable platform chemicals (Hick et al., 2010; Käldström et al., 2014; Schüth et al., 2014; Kaufman Rechulski et al., 2015). In comparison to the classical depolymerization of cellulose in acidic water, the activation energy barrier associated to the mechanocatalytic depolymerization was reduced by $66 \%$, highlighting the synergistic effect between mechanical forces and catalysis (Kaufman Rechulski et al., 2015). In addition, in contrast to the classical ball-milling of cellulose, the overall energy required for the mechanocatalytic depolymerization of cellulose is much lower thanks to much shorter reaction times (2-6 h, depending on the milling mode, vs. 24-48 h for classical ball-milling).

For an effective mechanocatalytic depolymerization of cellulose, the targeted catalyst must be mechanically robust, and possesses sites that are physically accessible and chemically active. Planetary mills, shaker mills, attrition mills, and rolling mills are few examples of mills that promote an intimate contact between catalysts and cellulose during mechanocatalysis processes (Schell and Harwood, 1994; Suryanarayana, 2001; Esteban and Carrasco, 2006; Bitra et al., 2009). Recently, Rinaldi and Schüth reported the mechanocatalytic depolymerization of cellulose in the presence of about $10 \mathrm{wt} \%$ of sulfuric acid (Schüth et al., 2014). Remarkably, about $90 \%$ of cellulose was converted to a water-soluble fraction, which is composed of low molecular weight oligosaccharides with a degree of polymerization in a window 1-7. One drawback associated to this pathway is the removal of sulfuric acid at the end of the mechanocatalytic process, which is a tedious step unless the resulting low molecular weight oligosaccharides are further processed through an acid-catalyzed reaction. Blair and co-workers reported that water-soluble products can be obtained in good yields $(\sim 70-80 \%)$ upon ball milling of cellulose in the presence of inorganic solid acids, particularly dealuminated kaolinite (Hick et al., 2010). It was observed that kaolinite caused a rapid depolymerization of cellulose thanks to its exfoliation during the milling. However, the selectivity to low molecular weight oligosaccharides was lower than in the case of $\mathrm{H}_{2} \mathrm{SO}_{4}$ due to the side formation of levoglucosane and brown colored chemicals, presumably humins or furanic derivatives (Hick et al., 2010; Shrotri et al., 2013). 
Recently, we reported that cellulose can be depolymerized to low molecular weight oligosaccharides by milling cellulose with Aquivion PW98, a strongly acidic perfluorinated sulfonic acid ionomer $\left(\mathrm{H}_{0}=-12\right.$, similar to $\left.\mathrm{H}_{2} \mathrm{SO}_{4}\right)$ (Karam et al., 2017). It was shown that Aquivion PW98 was chemically and mechanically resistant to the milling, permitting its long term recycling without altering its performances. In this article, we investigate the effect of the proton loading, stirring rate and Aquivion/cellulose mass ratio on the mechanocatalytic process. In particular, we point out the critical effect of water, even in trace amount, on the depolymerization rate of cellulose.

\section{MATERIALS AND METHODS}

\section{Reagents}

Microcrystalline cellulose (Avicel PH200, FMC Biopolymer) was utilized to investigate the performance of different solid catalysts.

\section{Catalyst Synthesis and Mechanocatalytic Depolymerization of Cellulose Catalyst Synthesis}

SBA-15- $\mathrm{SO}_{3} \mathrm{H}$ catalyst was prepared following a reported procedure (Karam et al., 2007). In a typical synthesis process, pluronic (4 g) was dissolved in $125 \mathrm{~g}$ of aqueous $\mathrm{HCl}(1.9 \mathrm{M})$ and stirred at room temperature. The solution was then heated at $40^{\circ} \mathrm{C}$ before addition of $7.7 \mathrm{~g}(0.0369 \mathrm{~mol})$ of TEOS. After stirring for $45 \mathrm{mn}$, MPTMS $(0.8 \mathrm{~g}, 0.0041 \mathrm{~mol})$ and $0.0369 \mathrm{~mol}$ of $35 \% \mathrm{H}_{2} \mathrm{O}_{2}$ was added. The solution was then stirred for $24 \mathrm{~h}$ at $40^{\circ} \mathrm{C}$ and aged into a teflon autoclave for an additional $24 \mathrm{~h}$ at $100^{\circ} \mathrm{C}$. The resulting solid was finally collected by filtration and thoroughly washed with water. The recovered $\mathrm{SBA}-15-\mathrm{SO}_{3} \mathrm{H}$ was dried in an oven at $50^{\circ} \mathrm{C}$ for $18 \mathrm{~h}$.

$\mathrm{CMK}-3-\mathrm{SO}_{3} \mathrm{H}$ was synthesized via a reported procedure by Jun et al. (2000). Typically, the calcined SBA-15 was impregnated with aqueous solution of sucrose containing sulfuric acid, $1 \mathrm{~g}$ of SBA-15 was added to a solution obtained by dissolving $1.25 \mathrm{~g}$ of sucrose and $0.14 \mathrm{~g}$ of $\mathrm{H} 2 \mathrm{SO} 4$ in $5 \mathrm{~g}$ of $\mathrm{H} 2 \mathrm{O}$. The mixture was placed in a drying oven for $6 \mathrm{~h}$ at $373 \mathrm{~K}$, and subsequently the oven temperature was increased to $433 \mathrm{~K}$ and maintained there for $6 \mathrm{~h}$. The sample turned dark brown or black during the treatment in the oven. The silica sample, containing partially polymerized and carbonized sucrose at the present step, was treated again at 373 and $433 \mathrm{~K}$ using the same drying oven after the addition of $0.8 \mathrm{~g}$ of sucrose, $0.09 \mathrm{~g}$ of $\mathrm{H} 2 \mathrm{SO} 4$, and $5 \mathrm{~g}$ of $\mathrm{H} 2 \mathrm{O}$. The carbonization was completed by pyrolysis with heating to typically $1173 \mathrm{~K}$ under vacuum. The carbon-silica composite obtained after pyrolysis was washed with $1 \mathrm{M} \mathrm{NaOH}$ solution ( 50 vol \% ethanol-50 vol \% H2O) twice at $373 \mathrm{~K}$ or $5 \mathrm{wt} \%$ hydrofluoric acid at room temperature, to remove the silica template. The template-free carbon product thus obtained was filtered, washed with ethanol, and dried at $393 \mathrm{~K}$. Thereafter, the recovered mesoporous carbon (so-called CMK-3) was suspended in concentrated $\mathrm{H}_{2} \mathrm{SO}_{4}$ ( $1 \mathrm{~g}$ of solid per $20 \mathrm{~mL}$ of acid) and stirred overnight. The $\mathrm{CMK}-3-\mathrm{SO}_{3} \mathrm{H}$ was washed several times with distilled water and then dried in an oven at $60^{\circ} \mathrm{C}$ overnight.

Aquivion PW66, PW79, PW87, and PW98 were used without further pretreatment as received from Solvay Specialty Polymers.

\section{Mechanocatalytic Depolymerization of Cellulsoe}

Various amounts of cellulose and catalyst were ground using a planetary ball-mill (Retsch MP100). The mixture of catalyst and cellulose were ground in a $125 \mathrm{~mL}$ bowl made of Zirconium Oxide, utilizing 20 of $10 \mathrm{~mm}$ balls made of the same material as the milling bowl. The experiments were performed at desired rate for a desired time as described in the main manuscript for each conditions investigated.

\section{Determination of Solubility}

After each milling, the milled mixture of cellulose and catalyst was recovered. The determination of solubility involved three parts, dispersion, filtration, and drying. The dispersion is carried out in a $20 \mathrm{~mL}$ flacon, weighing $300 \mathrm{mg}$ of the solid mixture and $20 \mathrm{~mL}$ distilled water, stirring and leaving it in an ultrasonic bath for $2 \mathrm{~h}$. The mixture is filtered through a $47 \mathrm{~mm}$ Millipore Pyrex Filter Holder; the PFTE filter has a pore size of $0.22 \mu \mathrm{m}$. The filter containing the solid after filtration is placed in a petri dish in the oven at $60^{\circ} \mathrm{C}$ overnight. The final mass is measured by the difference between the filter containing the dry solid at ambient temperature and the initial mass of the filter.

\section{RESULTS AND DISCUSSIONS}

Aquivion PW98, sulfonated SBA-15 and sulfonated mesoporous carbon $\left(\mathrm{CMK}-3-\mathrm{SO}_{3} \mathrm{H}\right)$ were either synthesized, or purchased, and initially screened as potential solid acid catalysts for the effective depolymerization of cellulose via ball-milling. More information on these solid acid catalysts are provided in the supporting information (SI). For starting experiments, $1 \mathrm{~g}$ of cellulose was mixed with $0.5 \mathrm{~g}$ of solid acid catalyst and stirred at $400 \mathrm{rpm}$ in a planetary ball-mill for $24 \mathrm{~h}$. The influence of experimental parameters such as the cellulose/catalyst mass ratio, stirring rate or reaction time is discussed later. The mechanocatalytic process is described in detail in the supporting information (section Introduction), and a schematic representation is shown in Scheme 1.

A $<5 \%$ solubility was observed when microcrystalline cellulose (MCC) was ball-milled for $24 \mathrm{~h}$ without any catalyst (Table 1, entry 1), in line with literature reported investigations (Meine et al., 2012). Treatment of neat MCC in a planetary ball mill in the presence of different solid acid catalysts remarkably improved the dissolution of the resulting product in water, showing the significance of intrinsic acid properties of solid acid catalysts in cleaving the $\beta-1,4$ glycosidic bonds in cellulose structures during the milling (Table 1). More information on the structure of products formed are provided at the end of the article.

Aquivion PW98 catalyst led to the highest MCC solubility in water ( $~ 80 \%)$ followed by CMK-3-SO $\mathrm{S}_{3} \mathrm{H}(87 \%)$ and $\mathrm{SBA}-\mathrm{SO}_{3} \mathrm{H}$ (60\%) (Table 1, entries 2-4). In our planetary ball-mill, kaolinite was found the least performant catalyst, leading to a product with a water solubility of only $50 \%$ (Table 1, entry 5). A significant difference of performance between the different tested Aquivion catalysts was observed (Table 1, entries 2, 6-8). The best result was obtained with Aquivion PW66 ( 99 \%) followed by PW98 (90\%), PW87 (80\%), and PW 79 (32\%). Interestingly, there is 


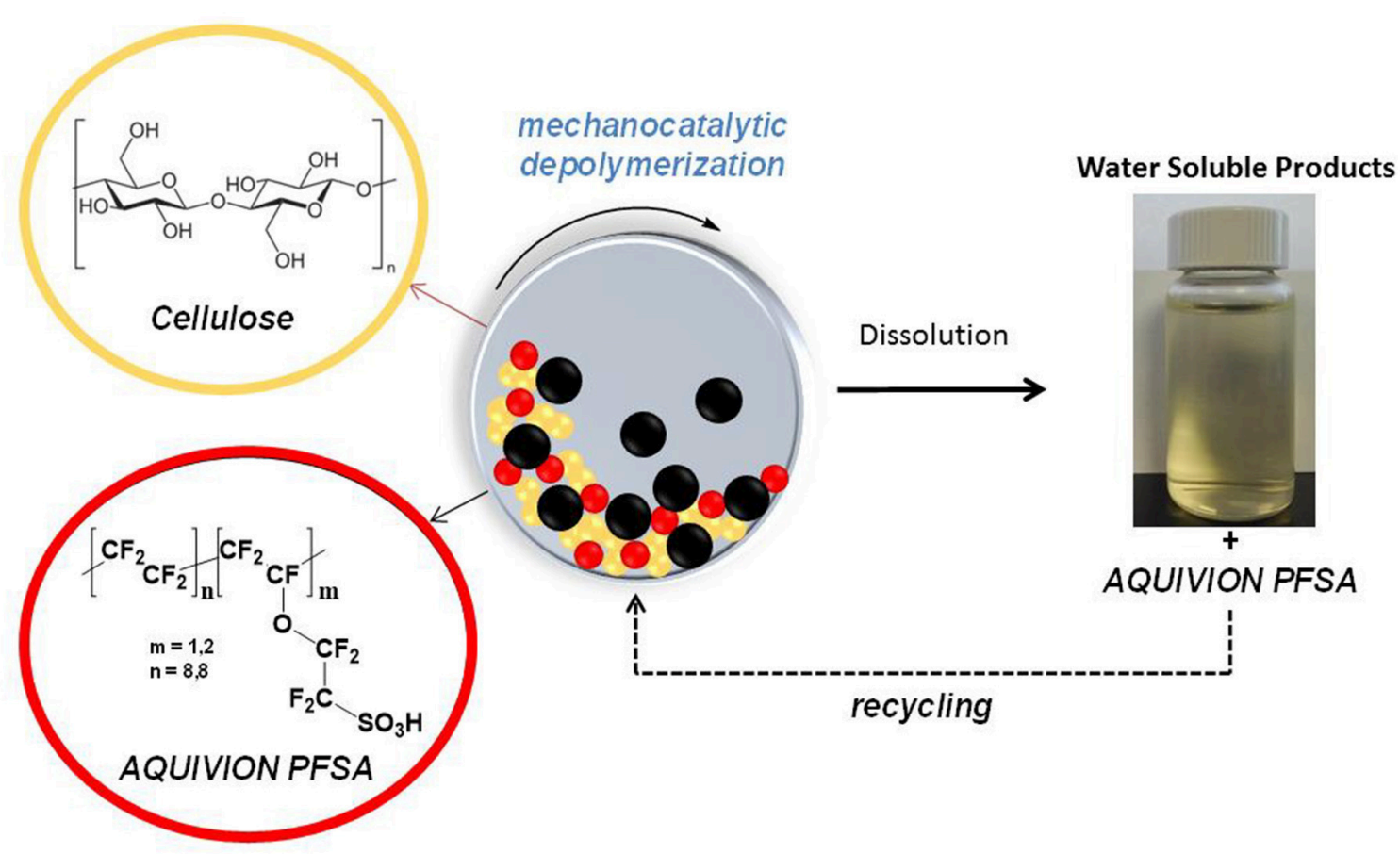

SCHEME 1 | Mechanocatalytic depolymerization of cellulose to water soluble oligomers.

TABLE 1 | Mechanocatalytic depolymerization of cellulose in the presence of different solid acid catalysts ${ }^{a}$.

\begin{tabular}{llcc}
\hline Entry & Catalyst & $\mathbf{H}^{+}$exchange capacity $(\mathbf{m m o l} / \mathbf{g})$ & Solubility (\%) \\
\hline 1 & Blank & - & $<5$ \\
2 & Aquivion PW98 & 1.0 & 90 \\
3 & SBA-SO $_{3} \mathrm{H}$ & 0.2 & 60 \\
4 & CMK-3-SO $_{3} \mathrm{H}$ & 0.7 & 87 \\
5 & Kaolinite (KGa-2) & - & 50 \\
6 & Aquivion PW66 & 1.45 & 99 \\
7 & Aquivion PW79 & 1.26 & 32 \\
8 & Aquivion PW87 & 1.15 & 80 \\
\hline
\end{tabular}

a Reaction conditions: Mass of Cellulose, 1 (g); Mass Catalyst, 0.5 (g); 400 rpm, Ball-milling time, $24 \mathrm{~h}$; 20 Zirconium Oxide-balls $d_{M B}=10 \mathrm{~mm} ;^{b} \pm 8 \%$.

no correlation between the proton loading of Aquivion samples and their efficiencies in the mechanocatalytic depolymerization of cellulose (Table 1, entries 2, 6-8). This result prompted us to investigate the humidity content of all the Aquivion catalysts used in this investigation, which will be discussed later in this manuscript.

Next, the effect of ball-milling time over the most active solid catalysts (Aquivion PW66 and PW98) identified in our preliminary investigations on the production of water soluble products from MCC was investigated. As shown in Figure 1, the solubility of ball-milled MCC increased markedly with ballmilling time, reaching a maximum of 90 and $99 \%$ within $24 \mathrm{~h}$ with Aquivion PW98 and PW66, respectively. Although both

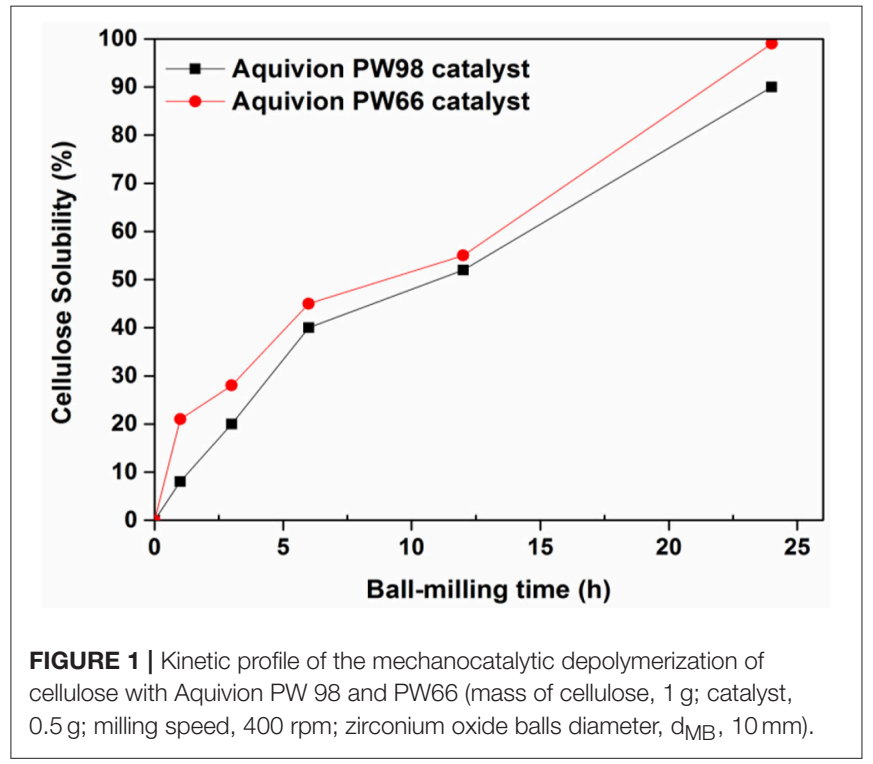

Aquivion samples have a different proton loading (Table 1), Aquivion PW66 and PW98 exhibited similar kinetic profiles, suggesting that the by- $\mathrm{SO}_{3} \mathrm{H}$ groups of Aquivion does not directly govern the kinetic of the reaction.

To support this hypothesis, the amount of cellulose was kept constant (1 g) while varying the amount of Aquivion PW98 (from 500 to $125 \mathrm{mg}$ ) during the ball-milling. It corresponds to a variation of the Aquivion PW98/cellulose mass ratio from 1:2 to 


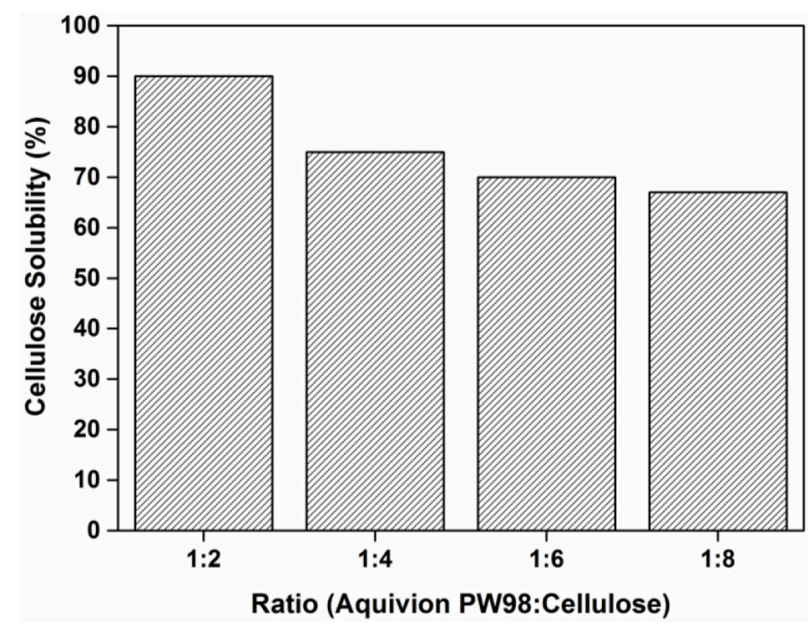

FIGURE 2 | Effect of the Aquivion PW98/cellulose mass ratio on the depolymerization rate of cellulose (mass of cellulose, $1 \mathrm{~g}$; milling speed, 400 rpm; zirconium oxide balls diameter $\mathrm{d}_{\mathrm{MB}}, 10 \mathrm{~mm}$; ball-milling time, $24 \mathrm{~h}$ ).

1:8 (Figure 2). Interestingly, the water solubility of MCC after the mechanocatalytic reaction did not differ dramatically between both assays, decreasing only from 90 to $70 \%$ upon moving from a 1:2 to a 1:8 Aquivion PW98/cellulose mass ratio, after $24 \mathrm{~h}$ of ball-milling. Altogether, these results strongly suggest that the depolymerization rate of cellulose under these conditions is not catalytically controlled and that mechanical forces probably play a more important role.

To further substantiate our assumption, the influence of the ball-milling speed in the process was also studied (Figure 3). During ball-milling, an increase in rotational speed leads to a subsequent rise in apparent energy due to the effective kinetic energy produced as a result of the frequent collisions of the balls between themselves and also between the balls and walls of the reactor. Working at a milling speed of $200 \mathrm{rpm}$ led to a solubility of $\sim 17 \%$ after $24 \mathrm{~h}$, which increased by more than 2 -folds (38\%) when the milling speed was further switched to $300 \mathrm{rpm}$ (Figure 3). At $400 \mathrm{rpm}$, a striking increase in solubility (90\%) was achieved. These results clearly demonstrate that the reaction kinetic is mostly governed by mechanical forces i.e., friction, collisions, shearing, etc, Nonetheless, when the milling speed was further increased to $500 \mathrm{rpm}$, a reduction of solubility was observed (from $90 \%$ at $400 \mathrm{rpm}$ to $77 \%$ at $500 \mathrm{rpm}$ ). At $500 \mathrm{rpm}$, colored tar-like insoluble products were formed leading to a decrease in solubility of cellulose after the mechanocatalytic process, due to excessive energy input in this case.

Assuming that mechanical forces have a strong impact on the depolymerization rate of cellulose, it occurred to us that the water content of Aquivion PFSA and cellulose will impact the mechanocatalytic process in a significant way, in particular by buffering the mechanical forces. Previously, it has been well-documented that the presence of a liquid, even in trace amount, dramatically impacts a mechanochemical process, a phenomenon known as liquid-assisted grinding (Käldström

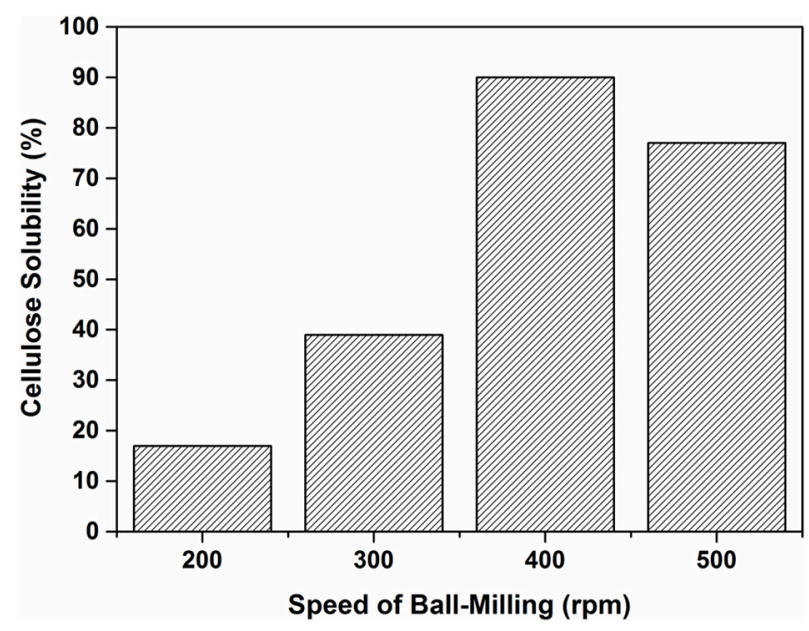

FIGURE 3 | Effect of the bowl stirring speed on the depolymerization rate of cellulose (mass of cellulose, $1 \mathrm{~g}$; Aquivion PW98, $0.5 \mathrm{~g}$; zirconium oxide balls diameter $\mathrm{d}_{\mathrm{MB}}, 10 \mathrm{~mm}$; ball-milling time, $24 \mathrm{~h}$ ).

et al., 2014). Aquivion PW98 and cellulose contained 7 and $6 \mathrm{wt} \%$ of water, respectively [i.e., $6.3 \mathrm{wt} \%$ of water for the Aquivion PW98/cellulose mixture (0.5:1)]. To assess the role of water, the mixture Aquivion PW98/cellulose was next freezedried before the mechanocatalytic process. The reaction was stopped after only $3 \mathrm{~h}$ of milling to clearly highlight the role of water. Remarkably, an increase in the mechanocatalytic depolymerization rate was observed and $55 \%$ of water soluble products were obtained after only $3 \mathrm{~h}$ of ball-milling vs. $20 \%$ without freeze-drying. An increase of the mechanocatalytic time from 3 to $6 \mathrm{~h}$ led to a nearly complete dissolution of cellulose (90\%). i.e., a reduction of the mechanocatalytic treatment time by 4 in comparison to non-freeze dried samples (Figure S1). Then, water was progressively added in order to monitor its effect on the milling. As shown in Figure 4, a sharp drop in the reaction product solubility was observed after $3 \mathrm{~h}$ of milling with aliquot amounts of $\mathrm{H}_{2} \mathrm{O}$, further stressing the important role played by water on the mechanocatalytic process.

When only Aquivion PW98 was freeze-dried, a slightly lower solubility of cellulose of $40 \%$ was observed (vs. 54\% for the freeze-dryied Aquivion PW98/cellulose mixture), indicating that water contained in cellulose was also impacting the mechanocatalytic process to some extent (Figure 5; details of water content estimation is provided in SI, section Materials and Methods). Aquivion PFSA PW98, PW66, PW87 have a similar water content of $7 \%$ and thus similarly behaved during the mechanocatalytic depolymerization of cellulose. In contrast, Aquivion PFSA PW79 has a water content of $22 \mathrm{wt} \%$ and was significantly less active than other Aquivion samples, providing a cellulose depolymerization product with a water solubility of only $32 \%$ after $24 \mathrm{~h}$ of ball-milling (Table 1). However, when Aquivion PW79 was freeze-dried before the mechanocataytic process, it behaved similarly than the other Aquivion samples ( $40 \%$ of cellulose was solubilized), further demonstrating the important role played by water (Figure 5). Here again, once 


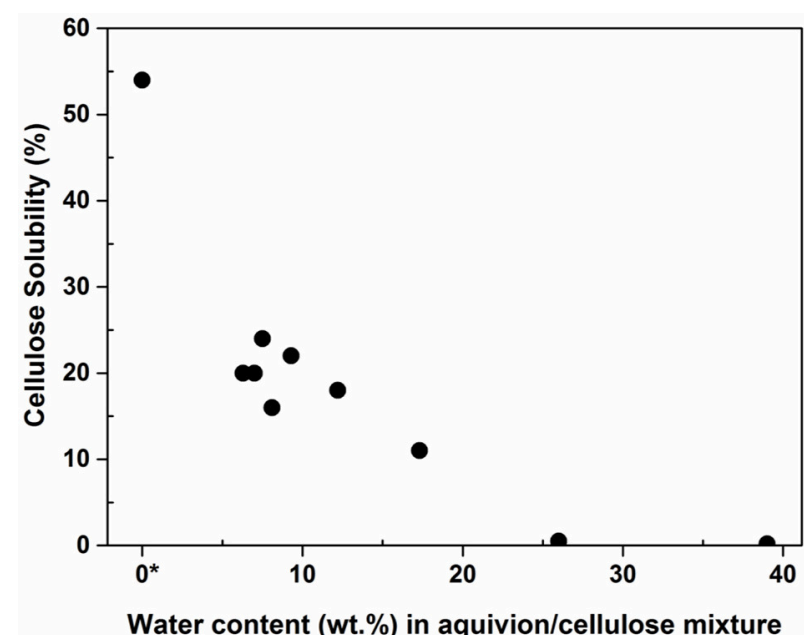

FIGURE 4 | Effect of water on the mechanocatalytic process. The value 0 was arbitrary fixed for the freeze-dried Aquivion PW98/cellulose mixture, although one should note that freeze-dried cellulose still contain chemically adsorbed water, difficult to measure.

freeze-dried, no significant difference of performance, in terms of depolymerization rate of cellulose, was observed between all Aquivion samples, although they have different proton loadings. These results confirm that the kinetics of the mechanocatalytic process is not controlled by the catalyst but mainly by mechanical forces, that can be tuned by the rotational stirring rate or the presence of water.

The water soluble products were analyzed in detail by mass spectrometry (MS), gas chromatography (GC) and high performance anionic-exchange chromatography with pulse amperometric detection (HPAEC-PAD). Consistently with previous reports using $\mathrm{H}_{2} \mathrm{SO}_{4}$ as an acid catalyst, Aquivion catalysts yielded oligosaccharides with a degree of polymerization (DP) up to 11 (MS). Monosaccharides accounted for $13 \%$ of the water soluble fraction, formed mainly by free D-glucose (96\%) and minor proportions of 1,6-anhydro-D-glucopyranose (levoglucosane, 3\%) and 1,5anhydro-D-glucofuranose (1\%) (GC; sample derivatization by sequential oximation-trimethylsilylation reactions as described in the SI). Disaccharides and oligosaccharides with DP 311 accounted for 21 and $65 \%$ of the water soluble product, respectively (Table 2). No oxidation or degradation product was detected, indicating that the mechanocatalytic process with Aquivion PW98 was fully selective to water soluble oligosaccharides. The disaccharide fraction was analyzed in depth using authentic commercially available standards (GC; sample derivatization by sequential oximation-acetylation reactions as described in the SI). All types of $\alpha / \beta$ positional regioisomers, namely $(1 \rightarrow 1)-,(1 \rightarrow 2)-,(1 \rightarrow 3)-,(1 \rightarrow 4)-$, and $(1 \rightarrow 6)$-linked glucobioses, were detected, with the $\beta$ - $(1 \rightarrow 4)$ linkage (cellobiose) being dominant $(79.5 \%$ of the disaccharide fraction). Considering that cellulose exclusively contains $\beta$ $(1 \rightarrow 4)$ glycosidic bonds, these results indicate that cellulose depolymerization during the mechanocatalytic process with

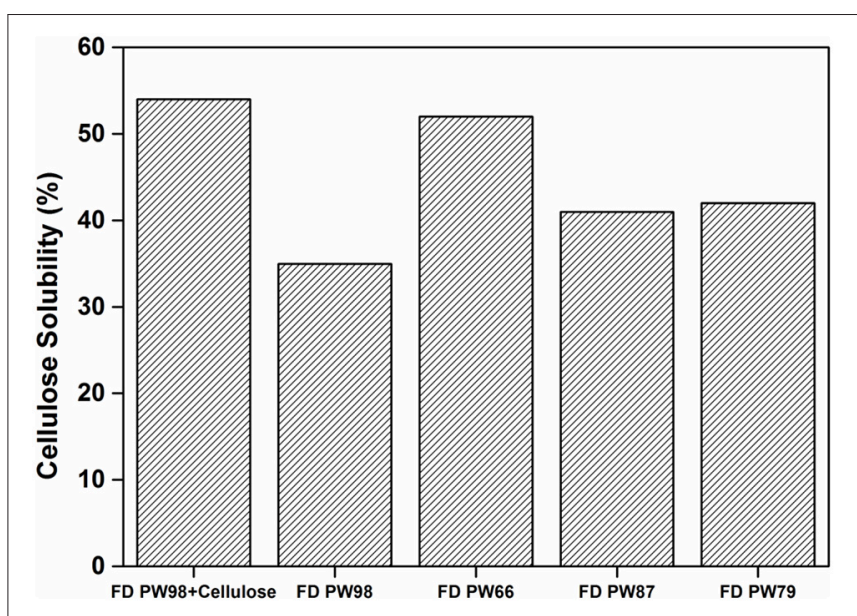

FIGURE 5 | Effect of freeze-drying of Aquivion samples on the mechanocatalytic process. FD, Freeze-dried.

Aquivion PW98 proceeds, to some extent, with concomitant self-glycosylation (reversion) reactions, in line with previous reports from Schüth and Beltramini (Meine et al., 2012; Shrotri et al., 2013).

In principle, cross-glycosydation reactions between free glucose and cello-oligosaccharides or between different cellooligosaccharides could lead to the formation of branched oligosaccharides. To assess the degree of branching, the mixture of oligosaccharides was subjected to a methylation $(\mathrm{MeI} / \mathrm{NaOH})$ (Ciucanu and Costello, 2003)-hydrolysis (TFA, $120{ }^{\circ} \mathrm{C}$ )deuteroboration $\left(\mathrm{NaBD}_{4}\right)$-acetylation $\left(\mathrm{Ac}_{2} \mathrm{O} / \mathrm{TFA}\right)$ sequence (Kim et al., 2006) prior to GC-MS analysis. This protocol affords the corresponding alditols labeled with deuterium at C1 , methylated at non-glycosylated positions and bearing acetyl groups at positions that were glycosylated in the starting oligosaccharide chain, which can be unequivocally assigned from the corresponding fragmentation patterns in MS by comparison with authentic standards (Sassaki et al., 2005). The data indicated that terminal and monoglycosylated residues accounted for more than $96 \%$ of the glucosyl units, the majority of the inner chain glucose units were glycosylated at position $\mathrm{O}-4$ as in cellulose ( $51.8 \%$ of total glucose). Only a small proportion (3.8\%) of the inner residues are doubly glycosylated at positions O-4 and O-6, supporting that most of the oligosaccharide material keep the $(1 \rightarrow 4)$-glycosylation pattern of the parent polysaccharide. Cellulose depolymerization is therefore the predominant reaction occurring under these conditions. Indeed, the HPAEC.PAD chromatogram obtained from the crude reaction material showed a profile compatible with the major presence of cello-oligosaccharides of increasing DP. Quantitative analysis indicated that over 93\% of the oligosaccharide material is comprised in the DP 2-to-6 fraction (Figure 6).

Noteworthy, the as-obtained oligosaccharides were highly hygroscopic. Water playing an important role in the reaction, the mechanocatalytic process was additionally performed under an argon atmosphere to assess a possible negative effect of 
TABLE 2 | Relative composition of oligosaccharides linkages recovered after mechanocatalytic depolymerization of cellulose with Aquivion PW98.

\begin{tabular}{|c|c|c|c|c|c|c|c|c|c|c|}
\hline \multicolumn{8}{|c|}{ Glycosidic bond (\%) } & \multicolumn{3}{|r|}{ Branching pattern (\%) } \\
\hline & & & & & & & & & & $\mathrm{OH}^{\sim 2} \mathrm{O}_{*}$ \\
\hline \multicolumn{2}{|c|}{$1 \rightarrow 4$} & \multicolumn{2}{|c|}{$1 \rightarrow 2$} & \multicolumn{2}{|c|}{$1 \rightarrow 3$} & \multicolumn{2}{|c|}{$1 \rightarrow 6$} & \multicolumn{2}{|c|}{$1 \rightarrow 1^{\prime}$} & $1 \rightarrow 4 \rightarrow 6$ \\
\hline \multicolumn{2}{|c|}{79.5} & \multicolumn{2}{|c|}{3} & \multicolumn{2}{|c|}{3} & \multicolumn{2}{|c|}{13} & \multicolumn{2}{|c|}{1.5} & 6 \\
\hline$\alpha$ & $\beta$ & $\alpha$ & $\beta$ & $\alpha$ & $\beta$ & $\alpha$ & $\beta$ & $\alpha, \alpha^{\prime}$ & $\alpha, \beta^{\prime}$ & - \\
\hline $6.5^{\mathrm{a}}$ & $73^{b}$ & $1.7^{\mathrm{C}}$ & $1.3^{d}$ & $2.3^{\mathrm{e}}$ & $0.7^{f}$ & $6.5^{\mathrm{g}}$ & $6.6^{\mathrm{h}}$ & $1^{i}$ & $0,5^{j}$ & - \\
\hline
\end{tabular}

${ }^{a}$ maltose; ${ }^{b}$ cellobiose; ${ }^{c}$ kojibiose; ${ }^{d}$ laminarabiose; ${ }^{e}$ nigerose; ${ }^{f}$ soforose; ${ }^{\text {isomaltose; }}{ }^{h}$ gentiobiose; ${ }^{i}$ trehalose; ${ }^{j}$ neotrehalose.

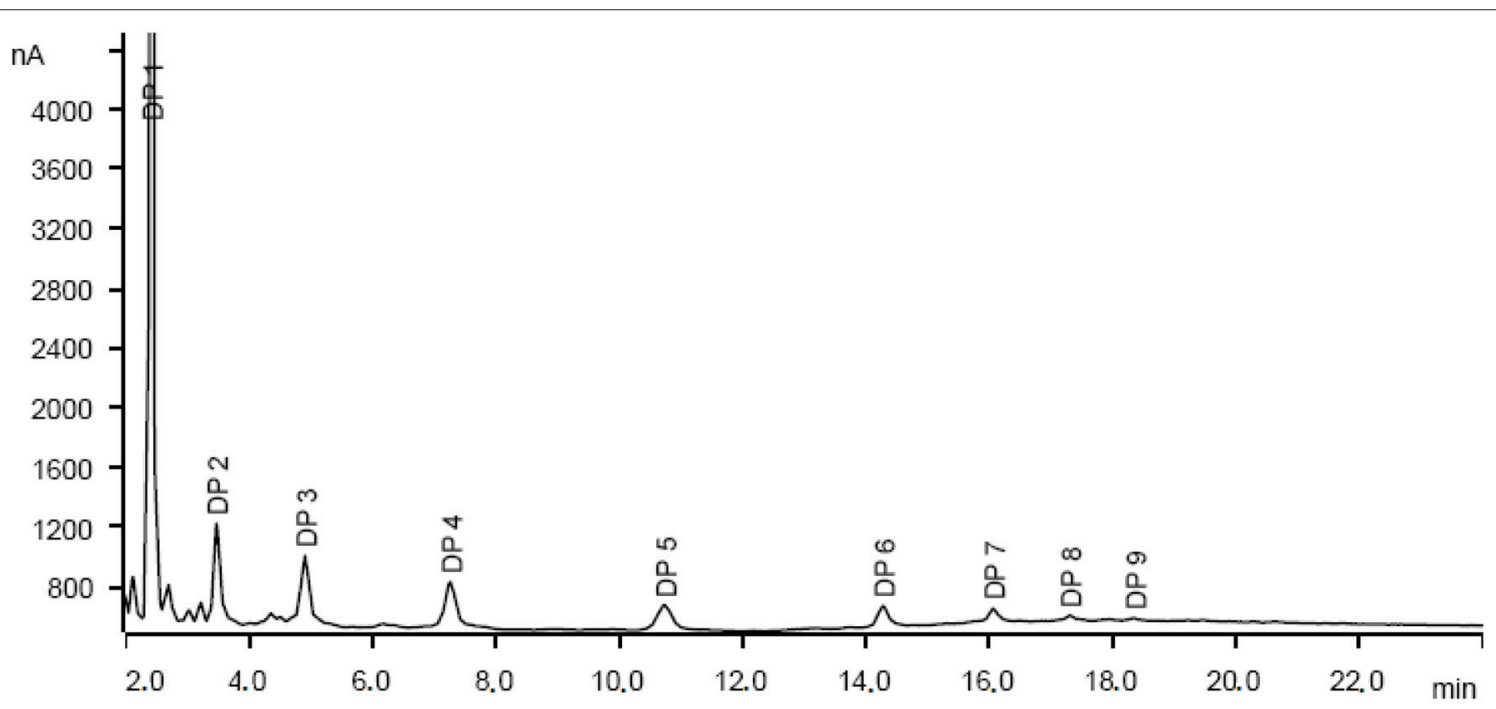

FIGURE 6 | HPAEC-PAC chromatogram (see SI for experimental description) of the oligosaccharide material obtained by the mechanocatalytic depolymerization of cellulose with Aquivion PW 98 (mass of cellulose, $1 \mathrm{~g}$; catalyst, $0.5 \mathrm{~g}$; milling speed, 400 rpm; zirconium oxide balls diameter, dMB, 10 mm; ball-milling time, 24 h).

water coming from the hygroscopicity of oligosaccharides during the milling. No significant change was observed as compared with the mechanocataytic process conducted under air, however, suggesting that mainly water initially contained in cellulose and Aquivion was responsible for the buffering of mechanical forces. This is an important point as regards industrial perspectives. Indeed, working in air will reduce the complexity of the system leading a more robust and lean process easier to be performed.

\section{CONCLUSIONS}

We have demonstrated that Aquivion, a sulfonated perfluorinated ionomer, was capable of promoting the selective depolymerization of cellulose to afford watersoluble oligosaccharides with a DP up to 11 . The detailed characterization of the water soluble fraction revealed that depolymerization and reversion reactions concomitantly occurred during the mechanocatalytic process, although the first largely predominated. The kinetic of the reaction is governed by mechanical forces, i.e., friction, collisions, shearing, etc. More importantly, we discovered that water contained in Aquivion and cellulose lowered the efficiency of the mechanocatalytic process, presumably by buffering mechanical forces. The plasticization effect of water in Aquivion is known although never reported in this frame. A removal of water before the mechanocatalytic process has permitted reducing the milling time. Under optimized conditions, yields of water soluble sugars of up to 90 and $97 \%$ were achieved using Aquivion PW98 and PW66, respectively. In comparison to $\mathrm{H}_{2} \mathrm{SO}_{4}$ which is commonly used in such application, Aquivion is easily separated from soluble sugars (dissolution of sugars in water and filtration of Aquivion), thus opening interesting perspectives to investigate the physicochemical properties of the as-obtained oligosaccharides or to further chemically process these sugars into specialty chemicals.

\section{AUTHOR CONTRIBUTIONS}

$\mathrm{AK}$ and PA performed the mechanocatalytic depolymerization of cellulose. KD and JG were in charge of the characterization 
of water soluble products obtained after the mechanocatalytic process. BE and SM were in charge of the critical role of water. $\mathrm{CO}$ was in charge of Aquivion catalysts (preparation, proton loading, water content, etc.). FJ supervized the work.

\section{ACKNOWLEDGMENTS}

Authors are grateful to the CNRS, the University of Poitiers, the Ministry of Research and the Région Nouvelle Aquitaine for their financial support. The International Consortium on Eco-conception and Renewable Resources (FR CNRS INCREASE 3707) and the chair TECHNOGREEN are also acknowledged for their funding. Authors are also grateful to the COST Action

\section{REFERENCES}

Barraud, E., Bégin-Colin, S., Le Caër, G., Barres, O., and Villiéras, F. (2008). Mechanically activated solid-state synthesis of hafnium carbide and hafnium nitride nanoparticles. J. Alloys Compd. 456, 224-233. doi: $10.1016 /$ j.jallcom.2007.02.017

Beyer, M. K., and Clausen-Schaumann, H. (2005). Mechanochemistry: the mechanical activation of covalent bonds. Chem. Rev. 105, 2921-2948. doi: $10.1021 / \mathrm{cr} 030697 \mathrm{~h}$

Bitra, V. S., Womac, A. R., Chevanan, N., Miu, P. I., Igathinathane, C., Sokhansanj, S., et al. (2009). Direct mechanical energy measures of hammer mill comminution of switchgrass, wheat straw, and corn stover and analysis of their particle size distributions. Powder Technol. 193, 32-45. doi: 10.1016/j.powtec.2009.02.010

Bozell, J. J., and Petersen, G. R. (2010). Technology development for the production of biobased products from biorefinery carbohydrates-the US epartment of energy's "Top 10" revisited. Green Chem. 12, 539-554. doi: 10.1039/B922014C

Carrasquillo-Flores, R., Käldström, M., Schüth, F., Dumesic, J. A., and Rinaldi, R. (2013). Mechanocatalytic depolymerization of dry (ligno) cellulose as an entry process for high-yield production of furfurals. ACS Catal. 3, 993-997. doi: $10.1021 / \mathrm{cs} 4001333$

Ciucanu, I., and Costello, C. E. (2003). Elimination of oxidative degradation during the per-O-methylation of carbohydrates. J. Am. Chem. Soc. 125, 16213-16219. doi: $10.1021 / \mathrm{ja} 035660 \mathrm{t}$

Climent, M. J., Corma, A., and Iborra, S. (2011). Converting carbohydrates to bulk chemicals and fine chemicals over heterogeneous catalysts. Green Chem. 13, 520-540. doi: 10.1039/c0gc00639d

Corma, A., Iborra, S., and Velty, A. (2007). Chemical routes for the transformation of biomass into chemicals. Chem. Rev. 107, 2411-2502. doi: 10.1021/cr050989d

Dhepe, P. L., and Fukuoka, A. (2008). Cellulose conversion under heterogeneous catalysis. ChemSusChem 1, 969-975. doi: 10.1002/cssc.200800129

Esteban, L. S., and Carrasco, J. E. (2006). Evaluation of different strategies for pulverization of forest biomasses. Powder Technol. 166, 139-151. doi: 10.1016/j.powtec.2006.05.018

Gallezot, P. (2012). Conversion of biomass to selected chemical products. Chem. Soc. Rev. 41, 1538-1558. doi: 10.1039/C1CS15147A

Groote, R., Jakobs, R. T., and Sijbesma, R. P. (2013). Mechanocatalysis: forcing latent catalysts into action. Polym. Chem. 4, 4846-4859. doi: $10.1039 / \mathrm{c} 3$ py00071k

Hick, S. M., Griebel, C., Restrepo, D. T., Truitt, J. H., Buker, E. J., Bylda, C., et al. (2010). Mechanocatalysis for biomass-derived chemicals and fuels. Green Chemistry 12, 468-474. doi: 10.1039/b923079c

Huber, G. W., Iborra, S., and Corma, A. (2006). Synthesis of transportation fuels from biomass: chemistry, catalysts, and engineering. Chem. Rev. 106, 4044-4098. doi: 10.1021/cr068360d

Jun, S., Joo, S. H., Ryoo, R., Kruk, M., Jaroniec, M., and Liu, Z., (2000). Synthesis of new, nanoporous carbon with hexagonally ordered mesostructure. J. Am. Chem. Soc. 122, 10712-10713. doi: 10.1021/ja002261e
FP1306 in supporting interaction between team members and for fruitful discussions on catalysis, processes, and cellulosic waste. JG thank the Spanish Ministerio de Economía y Competitividad (MINECO; Contract No. CTQ2015-64425-C2-1-R) and the Junta de Andalucía (Contract No. FQM2012-1467) for financial support. Co-financing from the European Regional Development Funds (FEDER and FSE) is also acknowledged.

\section{SUPPLEMENTARY MATERIAL}

The Supplementary Material for this article can be found online at: https://www.frontiersin.org/articles/10.3389/fchem. 2018.00074/full\#supplementary-material

Käldström, M., Meine, N., Farès, C., Schüth, F., and Rinaldi, R. (2014) Correction: deciphering 'water-soluble lignocellulose' obtained by mechanocatalysis: new insights into the chemical processes leading to deep depolymerization. Green Chem. 16, 4994-4994. doi: 10.1039/C4GC $90048 \mathrm{~K}$

Karam, A., De Oliveira Vigier, K., Marinkovic, S., Estrine, B., Oldani, C., and Jérôme, F. (2017). Conversion of cellulose into amphiphilic Alkyl glycosides catalyzed by aquivion, a perfluorosulfonic acid polymer. ChemSusChem 10, 3604-3610. doi: 10.1002/cssc.201700903

Karam, A., Gu, Y., Jérôme, F., Douliez, J.-P. and Barrault, J. (2007). Significant enhancement on selectivity in silica supported sulfonic acids catalyzed reactions. Chem. Commun. 2222-2224. doi: 10.1039/B702997G

Kaufman Rechulski, M. D., Käldström, M., Richter, U., Schüth, F., and Rinaldi, R. (2015). Mechanocatalytic depolymerization of lignocellulose performed on hectogram and kilogram scales. Indus. Eng. Chem. Res. 54, 4581-4592. doi: 10.1021/acs.iecr.5b00224

Kim, J. S., Reuhs, B. L., Michon, F., Kaiser, R. E., and Arumugham, R. G. (2006). Addition of glycerol for improved methylation linkage analysis of polysaccharides. Carbohydr. Res. 341, 1061-1064. doi: 10.1016/j.carres.2006.03.008

Klemm, D., Heublein, B., Fink, H. P., and Bohn, A. (2005). Cellulose: fascinating biopolymer and sustainable raw material. Angew. Chem. Int. Ed. 44, 3358-3393. doi: 10.1002/anie.200460587

Loerbroks, C., Rinaldi, R., and Thiel, W. (2013). The electronic nature of the $1,4-\beta$-glycosidic bond and its chemical environment: DFT insights into cellulose chemistry. Chem. A Eur. J. 19, 16282-16294. doi: 10.1002/chem. 201301366

Luterbacher, J., Alonso, D. M., and Dumesic, J. (2014). Targeted chemical upgrading of lignocellulosic biomass to platform molecules. Green Chem. 16, 4816-4838. doi: 10.1039/C4GC01160K

Meine, N., Rinaldi, R., and Schüth, F. (2012). Solvent-free catalytic depolymerization of cellulose to water-soluble oligosaccharides. ChemSusChem 5, 1449-1454. doi: 10.1002/cssc.201100770

Moon, R. J., Martini, A., Nairn, J., Simonsen, J., and Youngblood, J. (2011). Cellulose nanomaterials review: structure, properties and nanocomposites. Chem. Soc. Rev. 40, 3941-3994. doi: 10.1039/c0cs00108b

Rinaldi, R., and Schüth, F. (2009a). Acid hydrolysis of cellulose as the entry point into biorefinery schemes. ChemSusChem 2, 1096-1107. doi: $10.1002 /$ cssc. 200900188

Rinaldi, R., and Schüth, F. (2009b). Design of solid catalysts for the conversion of biomass. Ener. Environ. Sci. 2, 610-626. doi: 10.1039/b902668a

Sassaki, G. L., Gorin, P. A., Souza, L. M., Czelusniak, P. A., and Iacomini, M. (2005). Rapid synthesis of partially O-methylated alditol acetate standards for GC-MS: some relative activities of hydroxyl groups of methyl glycopyranosides on Purdie methylation. Carbohydr. Res. 340, 731-739. doi: 10.1016/j.carres.2005.01.020

Schell, D. J., and Harwood, C. (1994). Milling of lignocellulosic biomass. Appl Biochem. Biotechnol. 45, 159-168. doi: 10.1007/BF02941795 
Schmidt, R., Fuhrmann, S., Wondraczek, L., and Stolle, A. (2016). Influence of reaction parameters on the depolymerization of $\mathrm{H} 2 \mathrm{SO} 4$-impregnated cellulose in planetary ball mills. Powder Technol. 288, 123-131. doi: 10.1016/j.powtec.2015.11.002

Schüth, F., Rinaldi, R., Meine, N., Käldström, M., Hilgert, J., and Rechulski, M. K. (2014). Mechanocatalytic depolymerization of cellulose and raw biomass and downstream processing of the products. Cat. Today 234, 24-30. doi: 10.1016/j.cattod.2014.02.019

Shen, T., and Gnanakaran, S. (2009). The stability of cellulose: a statistical perspective from a coarse-grained model of hydrogen-bond networks. Biophys. J. 96, 3032-3040. doi: 10.1016/j.bpj.2008.12.3953

Shrotri, A., Lambert, L. K., Tanksale, A., and Beltramini, J. (2013). Mechanical depolymerisation of acidulated cellulose: understanding the solubility of high molecular weight oligomers. Green Chem. 15, 2761-2768. doi: $10.1039 / \mathrm{c} 3 g c 40945 \mathrm{~g}$

Siró, I., and Plackett, D. (2010). Microfibrillated cellulose and new nanocomposite materials: a review. Cellulose 17, 459-494. doi: 10.1007/s10570-010-9405-y

Suryanarayana, C. (2001). Mechanical alloying and milling. Prog. Mater. Sci. 46, 1-184. doi: 10.1016/S0079-6425(99)00010-9

Van de Vyver, S., Geboers, J., Jacobs, P. A., and Sels, B. F. (2011). Recent advances in the catalytic conversion of cellulose. ChemCatChem 3, 82-94. doi: $10.1002 /$ cctc. 201000302

Wang, J., Xi, J., and Wang, Y. (2015). Recent advances in the catalytic production of glucose from lignocellulosic biomass. Green Chem. 17, 737-751. doi: 10.1039/C4GC02034K
Yabushita, M., Kobayashi, H., and Fukuoka, A. (2014). Catalytic transformation of cellulose into platform chemicals. App. Cat. B 145, 1-9. doi: 10.1016/j.apcatb.2013.01.052

Zakrzewska, M. E., Bogel-Łukasik, E., and Bogel-Łukasik, R. (2010). Ionic liquid-mediated formation of 5-hydroxymethylfurfural- A promising biomassderived building block. Chem. Rev. 111, 397-417. doi: 10.1021/cr100171a

Zhang, Q., and Jérôme, F. (2013). Mechanocatalytic deconstruction of cellulose: an emerging entry into biorefinery. ChemSusChem 6, 2042-2044. doi: $10.1002 /$ cssc. 201300765

Zhou, C.-H., Xia, X., Lin, C.-X., Tong, D.-S., and Beltramini, J. (2011). Catalytic conversion of lignocellulosic biomass to fine chemicals and fuels. Chem. Soc. Rev. 40, 5588-5617. doi: 10.1039/c1cs15124j

Conflict of Interest Statement: The authors declare that the research was conducted in the absence of any commercial or financial relationships that could be construed as a potential conflict of interest.

Copyright (c) 2018 Karam, Amaniampong, García Fernández, Oldani, Marinkovic, Estrine, De Oliveira Vigier and Jérôme. This is an open-access article distributed under the terms of the Creative Commons Attribution License (CC BY). The use, distribution or reproduction in other forums is permitted, provided the original author(s) and the copyright owner are credited and that the original publication in this journal is cited, in accordance with accepted academic practice. No use, distribution or reproduction is permitted which does not comply with these terms. 